\title{
Lignin First: Confirming the Role of the Metal Catalyst in Reductive Fractionation
}

\author{
Lu Chen ${ }^{\S}$ Antoine P. van Muyden, ${ }^{\S}$ Xinjiang Cui, Zhaofu Fei, Ning Yan, Gabor Laurenczy,* \\ and Paul J. Dyson*
}

Cite This: JACS Au 2021, 1, 729-733

Read Online

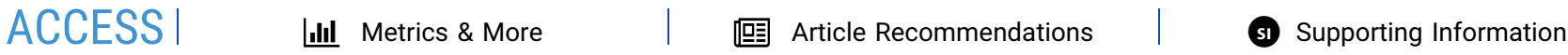

ABSTRACT: Rhodium nanoparticles embedded on the interior of hollow porous carbon nanospheres, able to sieve monomers from polymers, were used to confirm the precise role of metal catalysts in the reductive catalytic fractionation of lignin. The study provides clear evidence that the primary function of the metal catalyst is to hydrogenate monomeric lignin fragments into more stable forms following a solvent-based fractionation and fragmentation of lignin.

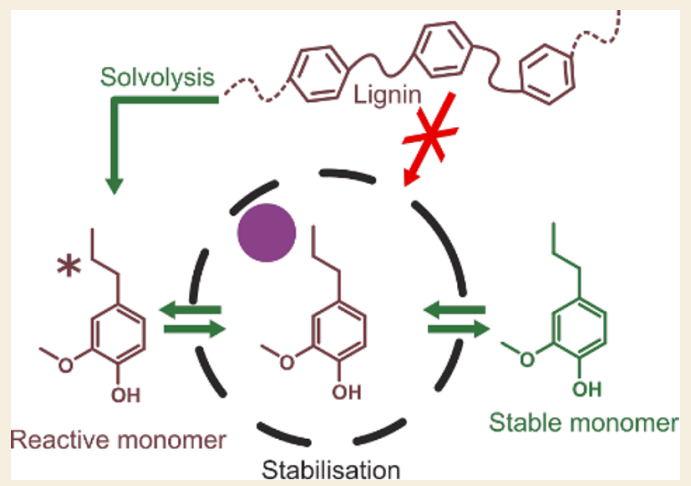

KEYWORDS: Reductive catalytic fractionation, Lignin first, Solvolysis, Rhodium, Sustainable chemistry

$\mathrm{R}$ eductive catalytic fractionation (RCF) is one of the key methods used in the so-called lignin-first approaches. ${ }^{1-7}$ In the presence of a metal catalyst and a reductant (usually $\mathrm{H}_{2}$ ), essentially all the $\beta-\mathrm{O}-4$ motifs can be cleaved in the native lignin present in a woody biomass, affording up to 50$60 \%$ of monomeric phenolic compounds. ${ }^{8-10}$ The transformation comprises three steps, including solvolysis fractionation of lignin from cellulose and hemicellulose by breaking the lignin-sugar linkages, followed by a fragmentation of polymeric lignin into monomers and oligomers via a $\mathrm{C}-\mathrm{O}$ bond cleavage, and finally stabilization of the reactive allyl species by hydrogenation. ${ }^{11,12}$ However, the exact role of the catalyst in RCF remains a matter of debate. ${ }^{13-15}$ Using a catalyst basket strategy, Sels et al. elegantly provided strong evidence that a protic solvent (e.g., $\mathrm{MeOH}$ ) is almost entirely responsible for both the fractionation and fragmentation of lignin. ${ }^{16}$ However, the basket used to confine the catalyst pellets consisted of 40 mesh ( $0.4 \mathrm{~mm}$ pore diameter), and therefore the possibility of lignin fragmentation over the metal catalyst in a solubilized polymeric form cannot be completely excluded. Indeed, other studies suggest that the metal catalyst contributes to breaking the ether linkages in lignin via hydrogenolysis. ${ }^{17-20}$ As a result of this uncertainty, many efforts to improve catalysts for RCF continue to be focused on enhancing $\beta$-O-4 bond cleavage in lignin model compounds, ${ }^{21-23}$ whereas other aspects might also deserve more attention.

To unambiguously address whether metal catalysts actively participate in the lignin depolymerization step or only play a role in the stabilization step, a catalyst encapsulated within a porous shell that prevents the lignin polymer from entering and interacting with the catalyst would be ideal (Scheme 1). Comparing the performance of the encapsulated metal catalyst with an exposed catalyst would provide a quantitative understanding of the contribution of the metal catalyst in RCF. Herein we chose to use Rh nanoparticles (NPs) embedded in porous hollow carbon spheres (Rh@HCS) to test this hypothesis (i.e., an efficient metal for hydrogenolysis confined within a porous carbon matrix). Although catalysts composed of $\mathrm{Pd}, \mathrm{Ru}$, and $\mathrm{Ni}$ are preferred for this reaction, $\mathrm{Rh}$ NPs were selected as a number of papers report their superior activity toward hydrogenolysis. ${ }^{24,25}$

The catalyst (termed Rh@HCS-Figure 1) was prepared by a modified solvothermal procedure (see the Supporting Information for details). ${ }^{26}$ Scanning electron microscopy (SEM) shows that the carbon spheres are uniform in size $(\sim 178 \mathrm{~nm})$ and thickness $(\sim 42 \mathrm{~nm})$ (Figure 1a). Transmission electron microscopy (TEM) shows that the Rh NPs are completely embedded within the interior of the carbon spheres and have a narrow size distribution $(\sim 1.4 \mathrm{~nm}$ average

Published: April 22, 2021

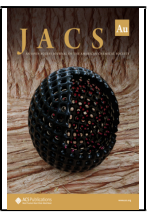


Scheme 1. Strategy Adopted to Probe the Mechanism of Reductive Catalytic Fractionation ${ }^{a}$

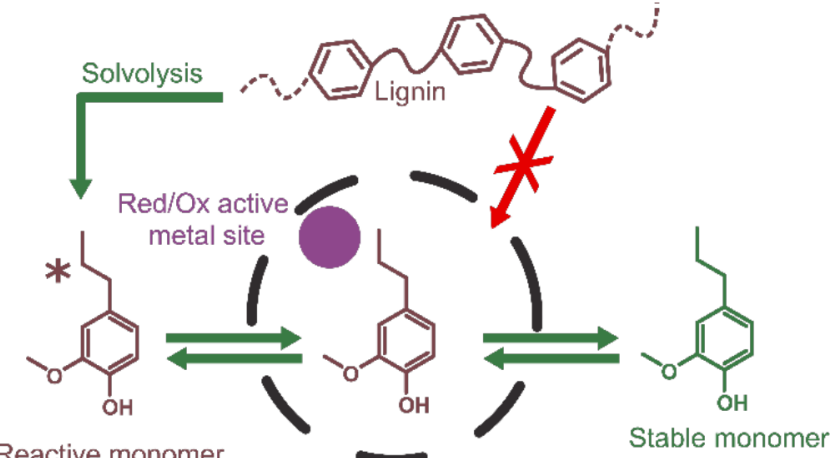

${ }^{a} 4-n$-Propyl guaiacol was used as a representative monomer. Lignin is depicted as a polymer composed of aromatic rings with the linkages, and methoxy groups are omitted for clarity.
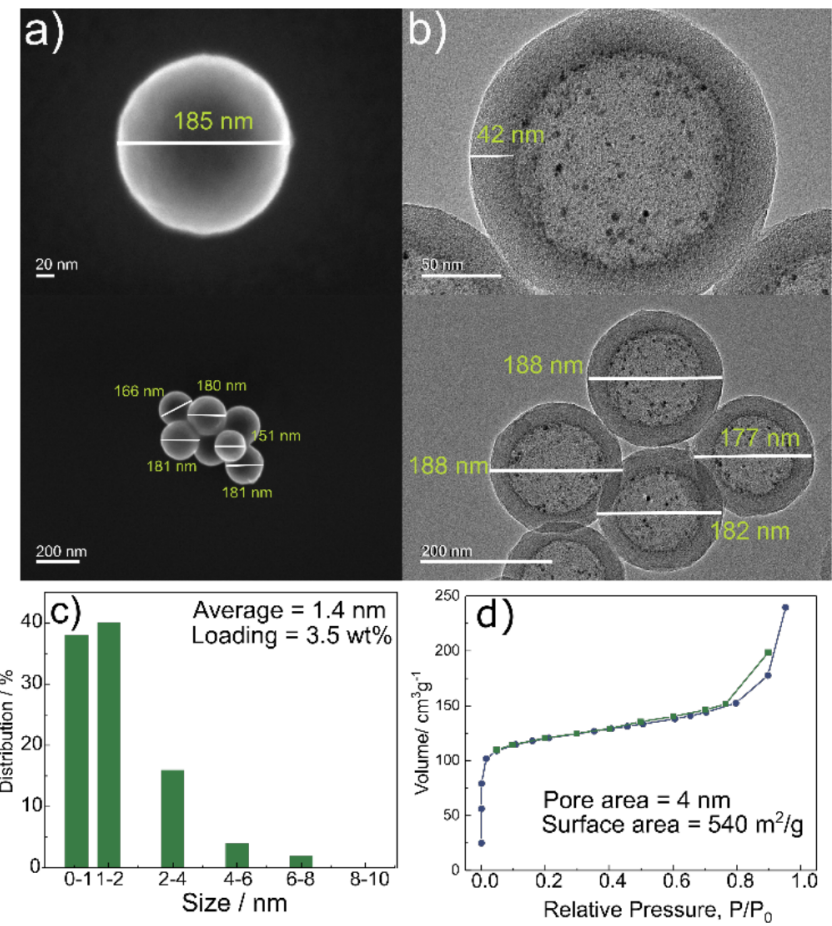

Figure 1. (a) SEM images of the Rh@HCS catalyst. (b) TEM images of the Rh@HCS catalyst. (c) Rh particle size distribution in the Rh@ HCS catalyst. (d) Physisorption curves. The relative pressure is $P / P_{\mathrm{o}}$, where $P_{\mathrm{o}}$ is the saturated vapor pressure of $\mathrm{N}_{2}$ at $-196{ }^{\circ} \mathrm{C}$.

diameter) (Figure $1 \mathrm{~b}$ ). The $\mathrm{Rh}$ loading was determined by inductively coupled plasma atomic emission spectroscopy (ICP-OES) as $3.5 \mathrm{wt} \%$. The $\mathrm{Rh}$ region of the $\mathrm{X}$-ray photoelectron spectroscopy (XPS) confirms the absence of $\mathrm{Rh}$ on the outer surface of the carbon spheres (Figure Sla-c), that is, exhibiting a broad carbon peak without any evidence of surface-bound Rh (Figure S1d). A physisorption analysis reveals a mesoporous material with a pore diameter of $4 \mathrm{~nm}$ and a surface area of $540 \mathrm{~m}^{2} / \mathrm{g}$ (Figure 1d). Commercial Rh/C was used as a control catalyst and was characterized by a TEM analysis (Figure S2) with the Rh NPs having a mean diameter of $5.7 \mathrm{~nm}$ unevenly distributed over the support material. Because of the larger size of the Rh NPs in the $\mathrm{Rh} / \mathrm{C}$ catalyst compared to the Rh@HCS catalyst, the Rh/C catalyst is expected to be less active, and to compensate for this a higher catalyst loading was used in the studies.

Polystyrene was used as a probe compound to determine whether the Rh NPs in the Rh@HCS catalyst can react with polymers. Polystyrene was chosen, as the $\mathrm{C}-\mathrm{C}$ bonds connecting the styrene cannot be cleaved, but the aromatic rings can be hydrogenated by $\mathrm{Rh}$ NPs. ${ }^{27}$ Polystyrene with three different molecular weights were tested, one low (1300 $\mathrm{g} / \mathrm{mol}$ ), one close to the molecular weight typical of lignin $(13000 \mathrm{~g} / \mathrm{mol})$, and a larger one $(30000 \mathrm{~g} / \mathrm{mol})$. The polystyrene was dissolved in tetrahydrofuran (THF) and reacted under forcing conditions to ensure hydrogenation in the presence of $\mathrm{Rh} \mathrm{NPs}$, that is, $200{ }^{\circ} \mathrm{C}, 4 \mathrm{MPa} \mathrm{H}_{2}, 15 \mathrm{~h}$. After the reaction, the solvent was removed, and Fourier-transform infrared (FT-IR) spectra were recorded (Figure 2 for 13000

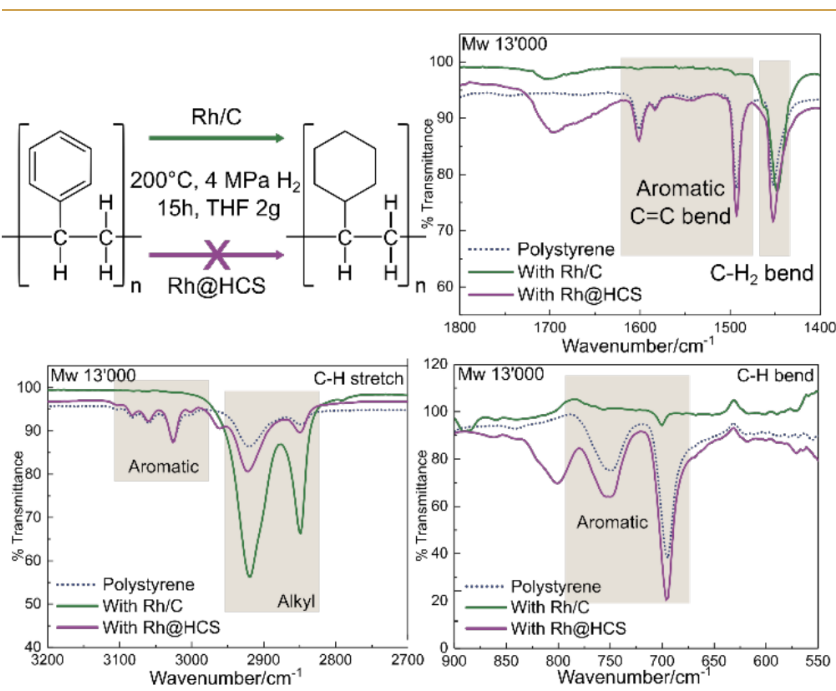

Figure 2. Relevant regions of the FT-IR spectra of polystyrene (Mw: $13000 \mathrm{~g} / \mathrm{mol}$ ) before and after a reaction with the Rh/C and Rh@ HCS catalysts. Pristine polystyrene-gray dots. Polystyrene after the reaction with the $\mathrm{Rh} / \mathrm{C}$ catalyst-green. Polystyrene after the reaction with the Rh@HCS catalyst-purple. Conditions: polystyrene (10 $\mathrm{mg}$ ), catalyst (Rh@HCS, 3.5 wt \%, $20 \mathrm{mg}$ or Rh/C, 5 wt \%, $14 \mathrm{mg}$.), THF $(2 \mathrm{~mL}), \mathrm{H}_{2}(4 \mathrm{MPa}), 200{ }^{\circ} \mathrm{C}, 15 \mathrm{~h}$.

g/mol polystyrene and Figure S3 for a comparison of all three polymers). The FT-IR spectra show the disappearance of the aromatic signal of the polymers from the reaction with the $\mathrm{Rh} /$ $C$ catalyst, whereas the FT-IR spectra remain unchanged when the Rh@HCS catalyst is used. The peaks corresponding to the $\mathrm{C}=\mathrm{C}$ bending vibrations $\left(1500-1600 \mathrm{~cm}^{-1}\right), \mathrm{C}-\mathrm{H}$ aromatic bending $\left(660-800 \mathrm{~cm}^{-1}\right)$, and $\mathrm{C}-\mathrm{H}$ stretches $(3000-3100$ $\mathrm{cm}^{-1}$ ) are highlighted in the figures to illustrate the differences. Thus, the Rh NPs encapsulated within the porous shell are unable to access the polystyrene, that is, the polystyrene cannot traverse the nanoscale shell, and it can be expected that lignin would also not be able to access the Rh NPs in the Rh@HCS catalyst. As a positive control, toluene was used as a substrate in place of polystyrene, and the toluene was hydrogenated to afford methyl cyclohexane in a near-quantitative yield by both catalysts under the same reaction conditions.

Birch sawdust was reacted in $\mathrm{MeOH}$ with $\mathrm{H}_{2}$ (4 MPa) at $200{ }^{\circ} \mathrm{C}$ in the presence of the Rh/C or Rh@HCS catalysts (see Figure S4 for temperature and pressure optimization), both affording monomers in $\sim 47 \%$ yield, confirming solvolysis is responsible for both the fractionation and fragmentation of 

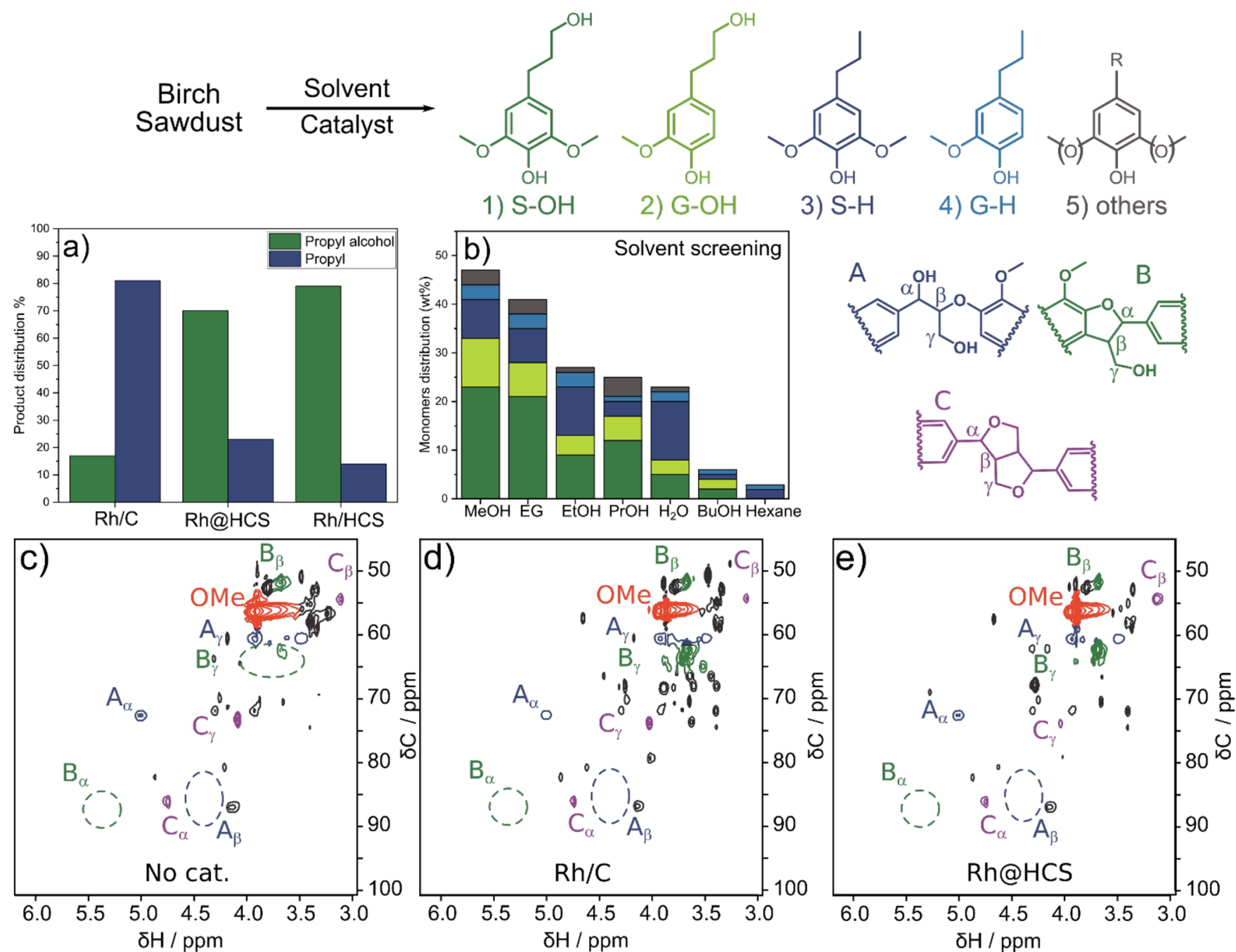

Figure 3. (a) Comparison between the Rh/C, Rh@HCS, and Rh/HCS catalysts under RCF conditions. (b) Protic solvent screening using Rh@ HCS catalyst. (c-e) 2D HSQC NMR spectra of the liquid products after RCF. (c) Without catalyst. (d) With the Rh/C catalyst. (e) With the Rh@HCS catalyst. Motifs are color-coded. $\beta$-O-4 labeled as A (in blue). Phenylcoumaran labeled as B (in green). Resinol labeled as C (in purple). Conditions: Rh@HCS (3.5 wt \%, $20 \mathrm{mg}$ ) or Rh/C (5 wt \%, $14 \mathrm{mg})$, sawdust (100 mg), MeOH (5 g), $\mathrm{H}_{2}(4 \mathrm{MPa}), 200{ }^{\circ} \mathrm{C}, 15 \mathrm{~h}$.

lignin. Analysis of the postreaction solution revealed a $\mathrm{Rh}$ content of $40 \mathrm{ppm}$, which would have a negligible influence on the reaction. Notably, there is a difference in the product distribution depending on the catalyst used (Figure 3a). The Rh@HCS catalyst affords more $n$-propyl alcohol monomers than $n$-propyl monomers, whereas the $\mathrm{Rh} / \mathrm{C}$ catalyst results in the opposite selectivity. This difference may be attributed to the support materials rather than the Rh NPs, with the Ndoped support in the Rh@HCS catalyst being less acidic than the pure carbon support in the $\mathrm{Rh} / \mathrm{C}$ catalyst. ${ }^{3,16,28}$ Therefore, a $\mathrm{Rh} / \mathrm{HCS}$ catalyst (Figure S5) was prepared with the $\mathrm{Rh}$ nanoparticles attached to the outer surface of the shell to remove the support effect. A similar product distribution was obtained to that of the Rh@HCS catalyst.

Two-dimensional heteronuclear single quantum coherence nuclear magnetic resonance (2D HSQC NMR) spectra of the liquid products obtained from both using catalysts and under catalyst-free conditions confirm that a cleavage of the $\beta$-O-4 motifs leads to a depolymerization via solvolysis as peaks corresponding to $A_{\beta}$ carbons are absent in all the spectra (Figure $3 \mathrm{c}-\mathrm{e}$; see Figure $\mathrm{S} 6$ for the whole spectra). The absence of peaks corresponding to $\mathrm{B}_{\alpha}$ carbons suggests that $\beta-5$ dimers are produced from the phenylcoumaran motifs in lignin. Notably, in the catalyst-free reaction, the peaks corresponding to $\mathrm{B}_{\gamma}$ carbons are of very low relative intensity, indicative of recondensation. All three carbons signals (i.e., $\mathrm{C}_{\alpha}$, $\mathrm{C}_{\beta}$, and $\mathrm{C}_{\gamma}$ ) corresponding to the resinol motifs are present. ${ }^{29}$

Common protic solvents used in RCF were also evaluated (Figure 3b), ${ }^{15}$ with longer-chain alcohols retarding the solvolysis, whereas an addition of another $\mathrm{OH}$ function increases it. Hexane was used as a nonprotic solvent with monomers obtained in only $2 \%$ yield, further confirming the mechanism.

In conclusion, we designed a catalyst, comprising Rh NPs immobilized within hollow nanoscale shells, to confirm the mechanism of RCF of biomass. Initially, we demonstrated that polymers cannot come into contact with the Rh NPs located at the interior of the hollow shell catalyst, whereas monomers can come into contact with the Rh NPs. The catalyst was then evaluated under typical RCF conditions and was shown to have a similar activity compared to a $\mathrm{Rh} / \mathrm{HCS}$ and $\mathrm{Rh} / \mathrm{C}$ catalyst, where the Rh NPs are exposed to all reagents, thus demonstrating that hydrogenolysis is not responsible for the cleavage of the $\beta$-O- 4 motif and providing unambiguous 
evidence to support this mechanism. ${ }^{11}$ The RCF involves initial solvolysis, occurring spontaneously under the harsh reaction conditions, followed by a stabilization of the reactive species generated via hydrogen by the Rh NPs.

\section{ASSOCIATED CONTENT}

\section{Supporting Information}

The Supporting Information is available free of charge at https://pubs.acs.org/doi/10.1021/jacsau.1c00018.

Experimental procedures, chemicals used, product analysis, analytical methods and data (PDF)

\section{AUTHOR INFORMATION}

\section{Corresponding Authors}

Gabor Laurenczy - Institute of Chemical Sciences and Engineering, Ecole Polytechnique Fedérale de Lausanne, 1015 Lausanne, Switzerland; 이이.org/0000-0002-43544521; Email: gabor.laurenczy@epfl.ch

Paul J. Dyson - Institute of Chemical Sciences and Engineering, Ecole Polytechnique Fedérale de Lausanne, 1015 Lausanne, Switzerland; (1) orcid.org/0000-0003-31173249; Email: pjd@epfl.ch

\section{Authors}

Lu Chen - Institute of Chemical Sciences and Engineering, École Polytechnique Fedérale de Lausanne, 1015 Lausanne, Switzerland

Antoine P. van Muyden - Institute of Chemical Sciences and Engineering, Ecole Polytechnique Fedérale de Lausanne, 1015 Lausanne, Switzerland

Xinjiang Cui - Institute of Chemical Sciences and Engineering, Ecole Polytechnique Fedérale de Lausanne, 1015 Lausanne, Switzerland

Zhaofu Fei - Institute of Chemical Sciences and Engineering, Ecole Polytechnique Fedérale de Lausanne, 1015 Lausanne, Switzerland

Ning Yan - Faculty of Engineering, University of Singapore, 119077, Singapore; 이이이.org/0000-0002-1877-9206

Complete contact information is available at:

https://pubs.acs.org/10.1021/jacsau.1c00018

\section{Author Contributions}

§(L.C. and A.P.v.M.) These authors contributed equally to this work.

Notes

The authors declare no competing financial interest.

\section{ACKNOWLEDGMENTS}

The authors thank the Swiss National Science Foundation and EPFL for financial support.

\section{ABBREVIATIONS}

RCF, (reductive catalytic fractionation); $\mathrm{MeOH}$, (methanol); NPs, (nanoparticles); Rh@HCS, (Rh nanoparticles (NPs) embedded in porous hollow car-bon spheres); ICP-OES, (inductively coupled plasma atomic emission spectroscopy); XPS, (X-ray photoelectron spectroscopy); TEM, (transmission electron microscopy); $\mathrm{Rh} / \mathrm{C}$, (commercial rhodium on carbon catalyst); THF, (tetrahydrofuran); FT-IR, (Fourier-transform infrared spectroscopy); Rh/HCS, (the Rh nanoparticles attached to the out surface of the carbon shell); 2D HSQC NMR, (two-dimensional heteronuclear single quantum coherence nuclear magnetic resonance)

\section{REFERENCES}

(1) Li, C.; Zhao, X.; Wang, A.; Huber, G. W.; Zhang, T. Catalytic Transformation of Lignin for the Production of Chemicals and Fuels. Chem. Rev. 2015, 115 (21), 11559-11624.

(2) Ragauskas, A. J.; Beckham, G. T.; Biddy, M. J.; Chandra, R.; Chen, F.; Davis, M. F.; Davison, B. H.; Dixon, R. A.; Gilna, P.; Keller, M.; Langan, P.; Naskar, A. K.; Saddler, J. N.; Tschaplinski, T. J.; Tuskan, G. A.; Wyman, C. E. Lignin Valorization: Improving Lignin Processing in the Biorefinery. Science (Washington, DC, U. S.) 2014, 344 (6185), 1246843.

(3) Narani, A.; Chowdari, R. K.; Cannilla, C.; Bonura, G.; Frusteri, F.; Heeres, H. J.; Barta, K. Efficient Catalytic Hydrotreatment of Kraft Lignin to Alkylphenolics Using Supported NiW and NiMo Catalysts in Supercritical Methanol. Green Chem. 2015, 17 (11), 5046-5057.

(4) Wong, S. S.; Shu, R.; Zhang, J.; Liu, H.; Yan, N. Downstream Processing of Lignin Derived Feedstock into End Products. Chem. Soc. Rev. 2020, 49 (15), 5510-5560.

(5) Korányi, T. I.; Fridrich, B.; Pineda, A.; Barta, K. Development of 'Lignin-First' Approaches for the Valorization of Lignocellulosic Biomass. Molecules 2020, 25 (12), 2815.

(6) Abu-Omar, M. M.; Barta, K.; Beckham, G. T.; Luterbacher, J. S.; Ralph, J.; Rinaldi, R.; Román-Leshkov, Y.; Samec, J. S. M.; Sels, B. F.; Wang, F. Guidelines for Performing Lignin-First Biorefining. Energy Environ. Sci. 2021, 14 (1), 262-292.

(7) Sun, Z.; Cheng, J.; Wang, D.; Yuan, T.; Song, G.; Barta, K. Downstream Processing Strategies for Lignin-First Biorefinery. ChemSusChem 2020, 13 (19), 5199-5212.

(8) Renders, T.; Van den Bossche, G.; Vangeel, T.; Van Aelst, K.; Sels, B. Reductive Catalytic Fractionation: State of the Art of the Lignin-First Biorefinery. Curr. Opin. Biotechnol. 2019, 56, 193-201.

(9) Sun, Z.; Fridrich, B.; De Santi, A.; Elangovan, S.; Barta, K. Bright Side of Lignin Depolymerization: Toward New Platform Chemicals. Chem. Rev. 2018, 118 (2), 614-678.

(10) Schutyser, W.; Renders, T.; Van Den Bosch, S.; Koelewijn, S. F.; Beckham, G. T.; Sels, B. F. Chemicals from Lignin: An Interplay of Lignocellulose Fractionation, Depolymerisation, and Upgrading. Chem. Soc. Rev. 2018, 47 (3), 852-908.

(11) Renders, T.; Van Den Bosch, S.; Koelewijn, S. F.; Schutyser, W.; Sels, B. F. Lignin-First Biomass Fractionation: The Advent of Active Stabilisation Strategies. Energy Environ. Sci. 2017, 10 (7), $1551-1557$

(12) Jing, Y.; Dong, L.; Guo, Y.; Liu, X.; Wang, Y. Chemicals from Lignin: A Review of Catalytic Conversion Involving Hydrogen. ChemSusChem 2020, 13 (17), 4181-4198.

(13) Anderson, E. M.; Stone, M. L.; Katahira, R.; Reed, M.; Beckham, G. T.; Román-Leshkov, Y. Flowthrough Reductive Catalytic Fractionation of Biomass. Joule 2017, 1 (3), 613-622.

(14) Song, Q.; Wang, F.; Cai, J.; Wang, Y.; Zhang, J.; Yu, W.; Xu, J. Lignin Depolymerization (LDP) in Alcohol over Nickel-Based Catalysts via a Fragmentation-Hydrogenolysis Process. Energy Environ. Sci. 2013, 6 (3), 994.

(15) Schutyser, W.; Van den Bosch, S.; Renders, T.; De Boe, T.; Koelewijn, S.-F.; Dewaele, A.; Ennaert, T.; Verkinderen, O.; Goderis, B.; Courtin, C. M.; Sels, B. F. Influence of Bio-Based Solvents on the Catalytic Reductive Fractionation of Birch Wood. Green Chem. 2015, 17 (11), 5035-5045.

(16) Van Den Bosch, S.; Renders, T.; Kennis, S.; Koelewijn, S. F.; Van Den Bossche, G.; Vangeel, T.; Deneyer, A.; Depuydt, D.; Courtin, C. M.; Thevelein, J. M.; Schutyser, W.; Sels, B. F. Integrating Lignin Valorization and Bio-Ethanol Production: On the Role of NiAl2O3catalyst Pellets during Lignin-First Fractionation. Green Chem. 2017, 19 (14), 3313-3326.

(17) Anderson, E. M.; Stone, M. L.; Hülsey, M. J.; Beckham, G. T.; Román-Leshkov, Y. Kinetic Studies of Lignin Solvolysis and 
Reduction by Reductive Catalytic Fractionation Decoupled in FlowThrough Reactors. ACS Sustainable Chem. Eng. 2018, 6 (6), 79517959.

(18) Chheda, J. N.; Román-Leshkov, Y.; Dumesic, J. A. Production of 5-Hydroxymethylfurfural and Furfural by Dehydration of BiomassDerived Mono- and Poly-Saccharides. Green Chem. 2007, 9 (4), $342-$ 350.

(19) Xu, H.; Yu, B.; Zhang, H.; Zhao, Y.; Yang, Z.; Xu, J.; Han, B.; Liu, Z. Reductive Cleavage of Inert Aryl C-O Bonds to Produce Arenes. Chem. Commun. 2015, 51 (61), 12212-12215.

(20) Wu, H.; Song, J.; Xie, C.; Wu, C.; Chen, C.; Han, B. Efficient and Mild Transfer Hydrogenolytic Cleavage of Aromatic Ether Bonds in Lignin-Derived Compounds over Ru/C. ACS Sustainable Chem. Eng. 2018, 6 (3), 2872-2877.

(21) Huang, X.; Ouyang, X.; Hendriks, B. M. S.; Gonzalez, O. M. M.; Zhu, J.; Korányi, T. I.; Boot, M. D.; Hensen, E. J. M. Selective Production of Mono-Aromatics from Lignocellulose over $\mathrm{Pd} / \mathrm{C}$ Catalyst: The Influence of Acid Co-Catalysts. Faraday Discuss. 2017, 202, 141-156.

(22) Hossain, M. A.; Phung, T. K.; Rahaman, M. S.; Tulaphol, S.; Jasinski, J. B.; Sathitsuksanoh, N. Catalytic Cleavage of the $\beta$-O-4 Aryl Ether Bonds of Lignin Model Compounds by Ru/C Catalyst. Appl. Catal., A 2019, 582, 117100.

(23) Zhang, B.; Qi, Z.; Li, X.; Ji, J.; Luo, W.; Li, C.; Wang, A.; Zhang, $\mathrm{T}$. $\mathrm{ReO}$ x /AC-Catalyzed Cleavage of $\mathrm{C}-\mathrm{O}$ Bonds in Lignin Model Compounds and Alkaline Lignins. ACS Sustainable Chem. Eng. 2019, 7 (1), 208-215.

(24) Ranade, V. S.; Prins, R. Hydrogenolysis of Benzylic Alcohols on Rhodium Catalysts. Chem. - Eur. J. 2000, 6 (2), 313-320.

(25) Chatterjee, M.; Chatterjee, A.; Ishizaka, T.; Kawanami, H. Rhodium-Mediated Hydrogenolysis/Hydrolysis of the Aryl Ether Bond in Supercritical Carbon Dioxide/Water: An Experimental and Theoretical Approach. Catal. Sci. Technol. 2015, 5 (3), 1532-1539.

(26) Wang, G. H.; Hilgert, J.; Richter, F. H.; Wang, F.; Bongard, H. J.; Spliethoff, B.; Weidenthaler, C.; Schüth, F. Platinum-Cobalt Bimetallic Nanoparticles in Hollow Carbon Nanospheres for Hydrogenolysis of 5-Hydroxymethylfurfural. Nat. Mater. 2014, 13 (3), 293-300.

(27) Maegawa, T.; Akashi, A.; Sajiki, H. A Mild and Facile Method for Complete Hydrogenation of Aromatic Nuclei in Water. Synlett 2006, 2006 (9), 1440-1442.

(28) Kasakov, S.; Shi, H.; Camaioni, D. M.; Zhao, C.; Baráth, E.; Jentys, A.; Lercher, J. A. Reductive Deconstruction of Organosolv Lignin Catalyzed by Zeolite Supported Nickel Nanoparticles. Green Chem. 2015, 17 (11), 5079-5090.

(29) Ralph, J.; Lundquist, K.; Brunow, G.; Lu, F.; Kim, H.; Schatz, P. F.; Marita, J. M.; Hatfield, R. D.; Ralph, S. A.; Christensen, J. H.; Boerjan, W. Lignins: Natural Polymers from Oxidative Coupling of 4Hydroxyphenyl- Propanoids. Phytochem. Rev. 2004, 3 (1-2), 29-60. 